\title{
A Kind of Improved Hybrid Algorithm for Vehicle Path Planning
}

\author{
Lingxia Liu $^{1}$ and Qiang Song ${ }^{2}$ \\ ${ }^{1}$ School of Software Engineering, Anyang Normal University, Anyang, China \\ ${ }^{3}$ School of Computer, Anyang Institute of Technology, Anyang, China \\ E-mail: aysq168@163.com
}

\begin{abstract}
Aiming to the requirements to find the shortest path among distribution nodes in logistics activities to guide the scheduling of logistics vehicles, this paper provides a new method which combines genetic algorithm (GA) and back propagation (BP) algorithm together for vehicle path planning to achieve the goal of saving transport cost. In this paper, the genetic algorithm is improved, and the shortcomings of the genetic algorithm are overcome, such as poor local search capability, easy to be early-maturing and not good enough global feasible solution. This hybrid algorithm covers the shortage of genetic algorithm efficiently, adopts the optimal preservation strategy in the genetic optimize operation, adopts the tournament selection method in the selection operation, to greatly improve the efficiency and function of the algorithm. Through the simulation to the performance of this improved hybrid algorithm for vehicle path planning based on genetic algorithm, and comparing with adaptive genetic algorithm and immune genetic algorithm, the results prove the advantages and effectiveness of the improved hybrid algorithm.
\end{abstract}

Keywords: Vehicle routing problem; genetic algorithm; back propagation; improved hybrid algorithm

\section{Introduction}

Vehicle Routing Problem (VRP) was proposed at the earliest by G. Danzig and J. Ramseur in 1959 [1], the problem is an important content in the link of logistics system optimization. The transportation vehicles are optimized and scheduled, selecting the best transport path of vehicles to reasonably arrange the vehicle scheduling order, can accelerate the response speed to customer's requirement, and effectively reduce the distance of the vehicle and service costs. In the areas such as postal delivery, vehicle route optimization, pipeline laying, and chain store delivery route optimization et al. numerous social domains, VRP has been widely used [2].

Because this problem belongs to the typical combinatorial optimization NP problem, it is difficult to be solved, and has extensive practical application background, thus for a long time a lot of scholars kept constantly research, exploration, researchers start from the initial accurate algorithm, step by step turn to wide heuristic algorithm field, such as genetic algorithm, tabu search algorithm, ant colony algorithm, etc. Yancheng Gong et al. put forward the method of transferring the time windows and vehicle capacity constraints on the minimum cost objective function to use genetic algorithm to solve the vehicle scheduling problem in the course of logistics distribution [3]; Chao proposed tabu search algorithm to solve VRP with multiple vehicle types [4]. Chen et al. studied the vehicle scheduling and routing selection problem in the logistics distribution of semi-trailer transport vehicle in steel field, and solved the problem through the genetic algorithm [5]; Shiquan Zhong et al. put forward the tabu search algorithm for VRP with soft and hard time window constraints to solving the problem [6]; Luliang Tang et al. proposed an improved ant colony algorithm aiming to the public transport path optimization problem 
with GPS data [7].These algorithms had made great achievements on solving VRP, but there are also some problems, such as tabu search algorithm has the shortcomings which has high dependence on the initial solution; Genetic algorithm has the disadvantages such as the local search ability is not strong, easy to trap into the premature, the overall quality of feasible solution is not very high.

Since genetic algorithm was proposed it had received the widespread attention, there were a lot of researchers who had applied the algorithm to solve the path optimization, task allocation and routing planning issues of wireless sensor network (WSN). For examples, Jingwen Tian et al. proposed the combination of genetic algorithm and ant colony algorithm to solve the problem of wireless sensor network path optimization [8].Meijuan Gao put forward an improved genetic algorithm and applied it to Web text mining [9]; Chengbo Li et al. proposed a kind of WMSNs multipath multi-objective optimization routing algorithm based on genetic algorithm [10]; Leilin et al. proposed a wireless sensor network path optimization algorithm based on genetic algorithm [11]; Kiabing Yang et al. put forward an improved Pareto fitness genetic algorithm to solve multi-objective combinatorial optimization problem [12]; Yangchu Yang et al. proposed a kind of near-space airship multidisciplinary optimization design program based on genetic algorithm design [13]; Xiaohua Li et al. put forward a kind of improved genetic algorithm for solving multiple-depot CARP problem [14];In addition, some improved genetic algorithm, such as adaptive genetic algorithm and immune genetic algorithm have been widely used. In many application fields, the genetic algorithm shows good performance, but genetic algorithm also has certain defects, such as in solving the large-scale problems, the convergence speed is slow, operation time is long, it's easy to fall into local optimal solution; the local search ability is poor, in the late evolution period the searching efficiency to exact solutions is very low and time-consuming; it is easy to cause premature convergence problem etc. in the practical application.

BP algorithm is a kind of basic intelligent algorithm, has the advantages of strong local optimization ability, easy to determine the optimal candidate solution of the precise location, the disadvantage is easy to fall into local extremum in the larger search space. Aiming at the defects of genetic algorithm and the advantage of BP algorithm, this paper proposes a kind of improved hybrid algorithm combing genetic algorithm and BP algorithm, these two kinds of algorithms can be complementary for each other and make the algorithm performance more better, thus, a kind of vehicle routing improved hybrid optimization algorithm is obtained to get a higher transport efficiency and more accurate operation result.

\section{VRP Mathematical Model}

The VRP mathematical model is expressed as follows [15]: there is a distribution center delivering to $\mathrm{k}$ customers, if the demand for goods at the $i$-th customer point is

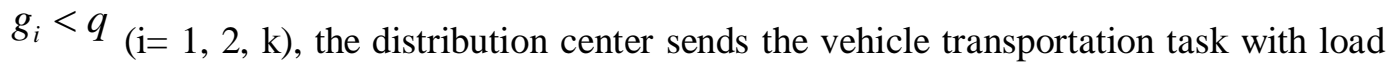
capacity q. supposing $g_{i}<q$, how to find the shortest paths to meet the demand of freight.

For effectively executing the route planning, in advance the number of vehicles needs to be estimated. In reality, the quantity demanded of vehicles depends on the complexity of cargo handling, if loading and unloading are more complex, the constraint conditions will be more, and the vehicle's actual cargo loading quantity will be less. The vehicle number selected by users themselves can be obtained by the following formula:

$$
n=\left[\sum g_{i} / \alpha q\right]+1
$$


Hereinto, $\mathrm{n}$ represents the needed vehicle number, [] represents the integer, $\alpha$ is a parameter, and $0<\alpha<1$. Under the situations with more constraints, if the loading and unloading are more complex, the $\alpha$ value is less, usually $\alpha=0.85$.

If ${ }^{c_{i j}}$ represents the route from point ${ }^{i}$ to point $j$, the number of distribution center is 0 , and the numbers of each customer point is $i(i=1,2, \ldots, k)$, the variables can be defined as follows:

The mathematics model of target functions can be built as follows.

$$
\begin{gathered}
x_{i j v}=\left\{\begin{array}{l}
1, \text { vehicle } v \text { travles from point } i \text { to point } j \\
0, \\
\text { others }
\end{array}\right. \\
x_{i j v}=\left\{\begin{array}{r}
1, \text { vehicle } v \text { is responsible to meet the demand of customer } i \\
0,
\end{array}\right. \\
\min l=\sum_{i=0}^{k} \sum_{j=0}^{k} \sum_{v=1}^{n} c_{i j} x_{i j v}
\end{gathered}
$$

The limitation conditions are as follows:

$$
\begin{gathered}
\sum_{i=1}^{k} g_{i} y_{i v} \leq q, \quad v=1,2, \ldots, n \\
\sum_{i=1}^{n} y_{i v}=1, \quad i=1,2, \ldots, k \\
\sum_{i=1}^{k} x_{i j v}=y_{j v}, \quad j=1,2, \ldots, k ; \quad v=1,2, \ldots, n \\
\sum_{i=1}^{k} x_{i j v}=y_{j v}, \quad i=1,2, \ldots, k ; \quad v=1,2, \ldots, n
\end{gathered}
$$

Expression (5) represents the total weight of freight loading should not exceed the maximum load for each vehicle; expression (6) is to ensure that the delivery task of each customer point can only be performed by one truck, and all transportation tasks are corporately completed by n trucks together; expressions (7) and (8) are to ensure the truck that arrived and left at a customer point have one and only one.

\section{The Improved Genetic Algorithm}

Genetic algorithm is a kind of new global optimization search algorithm, with its outstanding features such as simpleness, generalness, robustness, being suitable for parallel processing and wide application range, establishes its status as one of computational intelligence key. But genetic algorithm also has more disadvantages, the most obvious is the bad local search ability, which is not suitable for fine adjustment of candidate solutions, and it is difficult to determine the accurate position of the optimal solution rapidly. But BP algorithm's local optimization ability is stronger, which is easy to determine the optimal location of the candidate solution, and it is easy to fall into local extremum in the larger search space. Therefore combining genetic algorithm with BP algorithm integrates their respective advantages, that is, the global convergence of genetic algorithm and the local search rapidity of $\mathrm{BP}$ algorithm, are used to make up the disadvantages of two algorithms respectively. 
In this paper, the traditional genetic algorithm is improved, in the process of selection, crossover and mutation operation of genetic algorithm, the optimal preservation strategy is used, that is, while choosing operation the individuals will be ordered incrementally according to the fitness value, and then the 10\%individuals with the largest fitness value in parent population will be directly taken as the individuals in the next generation of population. This method can guarantee the best individuals obtained in the genetic process won't be damaged by crossover and mutation operation, keep the optimal individual to the next generation; meanwhile, the strategy can maintain the diversity of their offspring. In the process of crossover and mutation operation, the optimal preservation strategy only keeps the best individuals, that is, only the child individuals whose result of crossover and mutation is better than that of the parents, the optimal preservation strategy can make local optimal individuals not easy to be eliminated, so as to enhance the global search ability of the algorithm.

(1) Selection. This paper gives up commonly used roulette strategy, adopts the tournament selection method for parameters selection, namely at first randomly chooses a certain number (Tour) of individuals from the population for comparing, the individuals with the highest fitness value will be selected as the parent chromosomes of next generation. Clearly, this selection mode also makes the individuals with higher fitness value have great "survival" opportunity. At the same time, because it only uses the relative value of fitness value as the choice standard, and isn't directly proportional with the size of fitness value, thus it can avoid the influence of the super individuals, to some extent to avoid the occurrence of the premature convergence and stagnation phenomenon.

In the vehicle route planning, the chromosomes are different paths, the population is a collection of multiple chromosomes, and the fitness value takes the reciprocal of the path length 1 . When using tournament selection strategy, the shorter is the path length 1 , the higher is the fitness value of chromosome, and the chromosomes with the highest fitness value are preserved as the parent chromosomes of next generation of populations. The parameter of tournament selection is the competition scale Tour, its scope is $[2$, Nind $]$.

The intensity of selection is:

$$
\text { Sel } \ln t_{\text {Tour }}(\text { Tour })=\sqrt{2[l v(\text { Tour })-l b \sqrt{4.14(\text { Tour })}]}
$$

The diversity loss is:

$$
\operatorname{LossDiv}_{\text {Tour }}(\text { Tour })=\text { Tour }^{-[1 /(\text { Tour }-1)]}-\text { Tour }^{-[\text {Tour } /(\text { Tour }-1)]}
$$

While Tour $=5$, the diversity loss is about $50 \%$.

The selection of variance is:

$$
\text { SelVar }_{\text {Tour }}(\text { Tour } 0=1-0.096 l b(1+7.11(\text { Tour }-1))
$$

Hereinto, SelVarTour(2) $=1-1 / \pi$.

(2) Mutation. Mutation operation in genetic algorithm is an important way to maintain the diversity of the species, which is not only regarded as the means to find the lost good genes because of the crossover operation, but also the powerful tool exploring the adjacent space and optimizing performance. The individuals of new population produces change with small probability or step size, the probability of variable transformation or step size is inversely proportional to the dimension (i.e., the number of variables), has nothing to do with the population size. In variation operation, the mutation rate should not be too big, if the mutation rate is greater than 0.5 , the genetic algorithm is degraded as random search, the search ability is lost.

The selection of variable step size is difficult, the optimal step length depends on the specific situation, even can be changed in the optimization process. This article adopts the mutation operator of the following: 
Considering the actual situation of the vehicle path planning problem, generally the form of natural number coding is adopted.

$$
X^{\prime}=x \pm 0.5 L \Delta
$$

In the expression, $\Delta=\sum_{i=0}^{m=1}\left[a(i) / 2^{i}\right], a(i)$ takes value 1 with probability $1 / m$, takes value 0 with probability $1-1 / m$, in this paper, $m=20$; $\mathrm{L}$ is the value range of the variable; $X$ is the variable value before mutation; $X^{\prime}$ is the variable value after mutation.

(3) Crossover. Because in the vehicle path planning problem the natural number coding is generally adopted, thus in the crossover process discrete crossover method is adopted, that is, the variable value is transformed among individuals, each variable of child individual can randomly select parent individuals with equal probability.

\section{BP Algorithm}

The basic idea of the hybrid algorithm proposed in this paper is combining genetic algorithm with BP algorithm together, integrating their advantages and making up their shortcomings. Here the basic principle of BP algorithm is simply introduced. The multilayer perceptron neural network model using BP algorithm is called BP neural network, which is composed of input layer, hidden layer and output layer. The learning process of BP algorithm consists of signal forward propagation and error back propagation. In forward propagation, the incoming sample is input from the input layer, and sent to output layer after the processing of each hidden layer step by step. If the actual output of output layer is inconsistent with the desired output, then turns to the error back propagation phase. The error back propagation is sending the output error in some form to the input layer via back propagation through the hidden layer, and the error is shared with all elements of each layer so as to get the error signals of each layer unit, the error signal is used as the basis for correcting the weight of each unit. The process of weight adjustment in each layer of signal forward propagation and error back propagation is carried out repeatedly. The process that weight constantly adjusts is the study and training process of the network.

This process executes continuously until the network output error reduces to an acceptable level, or reaches at the preset times of learning.

$\mathrm{BP}$ algorithm's learning rule is based on the minimum mean square error criterion, the steps are as follows:

Initialize the weights. $\omega_{s p}=\operatorname{Random}(\wedge), s p_{\text {is }} i j, j k$ or $k l$.

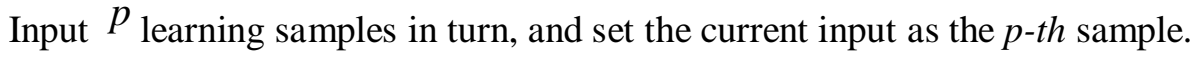

Calculate the output of each layer. $x_{j}^{\prime \prime}, x_{k}^{\prime \prime}$ and $y_{l}, j=0,1,2, \ldots, n_{1} ; k=0,1,2, \ldots, n_{2} ; l=0,1,2, \ldots, m-1$.

Calculate the back propagation of error.

$$
\begin{gathered}
\delta_{k l}^{(p)}=\left(d_{l}^{(p)}-y_{l}^{(p)}\right) y_{l}^{(p)}\left(1-y_{l}^{(p)}\right), \quad l=0,1,2, \ldots, m-1 \\
\delta_{j k}^{(p)}=\sum_{l=0}^{m-1} \delta_{k l}^{(p)} \omega_{k l}^{\prime \prime} x_{k}^{\prime(p)}\left(1-x_{k}^{((p)}\right), k=0,1,2, \ldots, n_{2} \\
\delta_{i j}^{(p)}=\sum_{l=0}^{m-1} \delta_{j k}^{(p)} \omega_{j k}^{\prime \prime} x_{j}^{(p)}\left(1-x_{j}^{(p)}\right), j=0,1,2, \ldots, n_{1}
\end{gathered}
$$

Record the values of each $x_{k}^{\prime(p)}, x_{j}^{\prime(p)}$ and $x_{i}^{(p)}$. 
(5) Record the sample's number $\mathrm{p}$ which has been studied. If $p<P$, then go to step (2) to continue to calculate, if $p=P$ then go to step (6).

(6) According to the weight correction formula to correct the weight of each layer.

(7) According to the new weights to calculate $x_{j}^{\prime}, x_{k}^{\prime \prime(p)}, y_{l}$ and $E_{T}$, if every $p$ and $l$ meet the expression $E_{T}<\varepsilon$ or reach the maximum times of learning, then stop learning, otherwise go to step (2) to continue a new round of study.

\section{The Implementation Steps of Improved Hybrid Algorithm}

Genetic algorithm and BP algorithm may have a variety of combination methods, the basic idea of the hybrid algorithm proposed in this paper is to use genetic algorithm to find the global optimal solution, in the adjacent optimization process between two times, the difference of mean square error is no longer meaningfully changing, at this time the solution has been fully close to the optimal solution, on the basis BP algorithm is used to fine-tune again until it meets the assessment requirements.

The implementation steps of proposed hybrid algorithm are as follows:

(1) Generate the initial population. Intersections are taken as nodes, each node's location is numbered, randomly generate some paths to do genetic optimization and produce a population, that is, a solution set, the solutions of the set are done individual evaluation to keep the solutions with high fitness, at the same time the solution set is locally optimized in BP algorithm to keep the solutions with high fitness, the solutions generated in two methods form into new population and continue the genetic optimization, until the optimization solution is generated to meet the requirements of the GA fitness value.

(2) The optimized solution meeting the requirements of GA fitness value are continuously to do optimally fine-tuning using $\mathrm{BP}$ algorithm to obtain the optimal solution conforming to the actual requirements. In this paper, the proposed hybrid algorithm actually combines BP algorithm with genetic algorithm for twice, the first time is when generating new population to find a local optimal solution, the second combination is used for fine-tuning to the optimization solution that the genetic algorithm produces. Figure 1 is the flow chart of the improved hybrid algorithm.

\section{The Analysis of Simulation Results}

In the simulation, the nodes number is 29 that need to be traversed by a selected truck, that is $k=29$, plus the distribution center there are total 30 points. Based on the MATLAB environment this paper respectively does simulation to adaptive genetic algorithm, immune genetic algorithm and improved hybrid algorithm, in order to compare and analyze the performance of the proposed hybrid algorithm in this paper.

In the MATLAB simulation environment, the selected 30 points coordinates respectively are $(87,7),(91,38),(83,46),(71,44),(64,60),(68,58),(83,69),(87,76)$, $(74,78),(71,71),(58,69),(54,62),(51,67),(37,84),(41,94),(2,99),(7,64),(22,60)$, $(25,62),(18,54),(4,50),(13,40),(18,40),(24,42),(25,38),(41,26),(45,21),(44,35)$, $(58,35),(62,32)$. Supposing $(87,7)$ represents the distribution center, the number is 0 , the follows are ordered according to the sequence. 


\subsection{The Simulation Experiment}

(1) The iteration times is set in simulation is 1000 times when the iteration times are same; respectively three kinds of algorithms are done simulation, to compare the path length. Figure 2, Figure 3, and Figure 4, respectively are simulation path diagrams obtained by three algorithms.

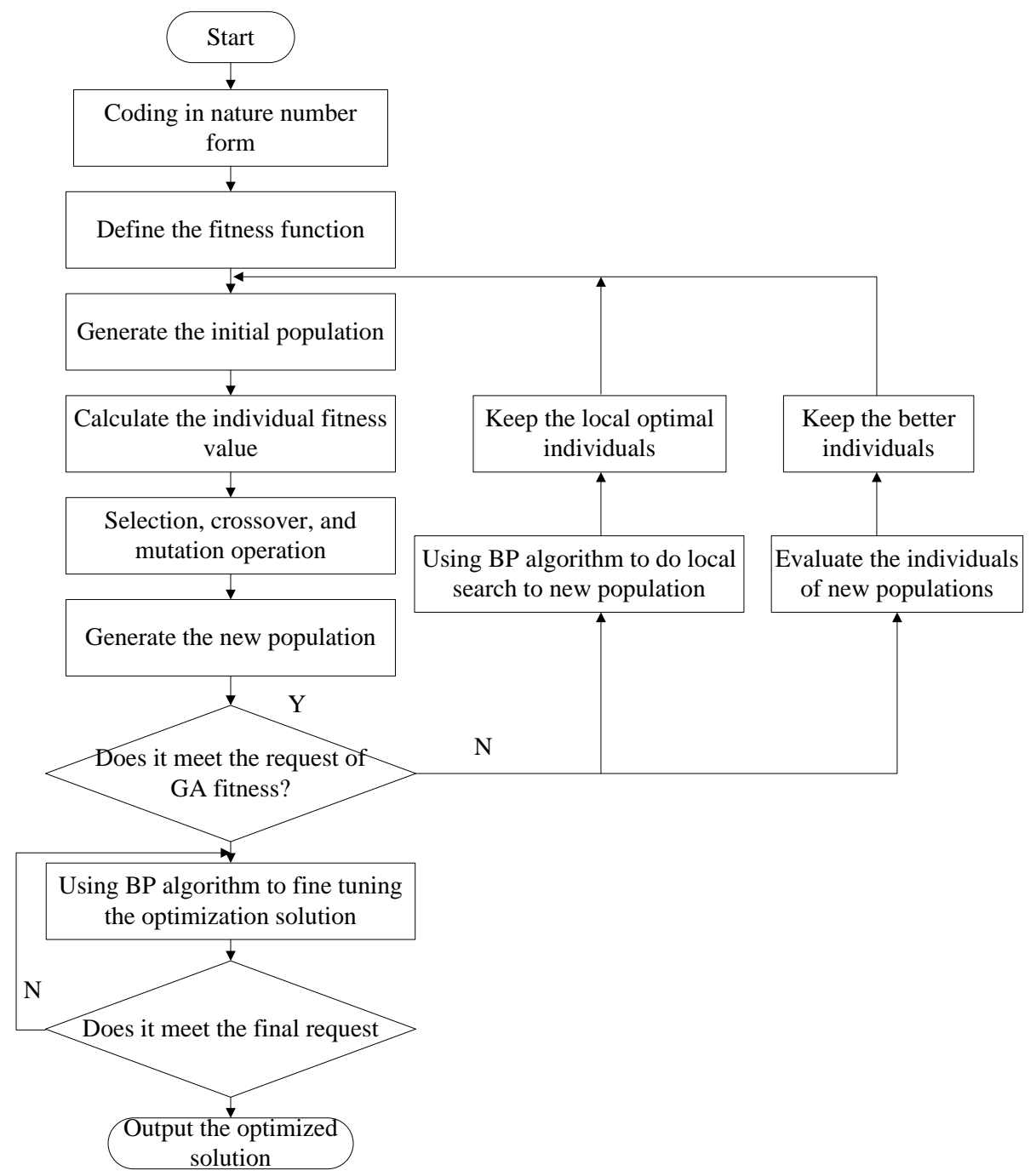

Figure 1. The Flowchart of Improved Hybrid Algorithm 


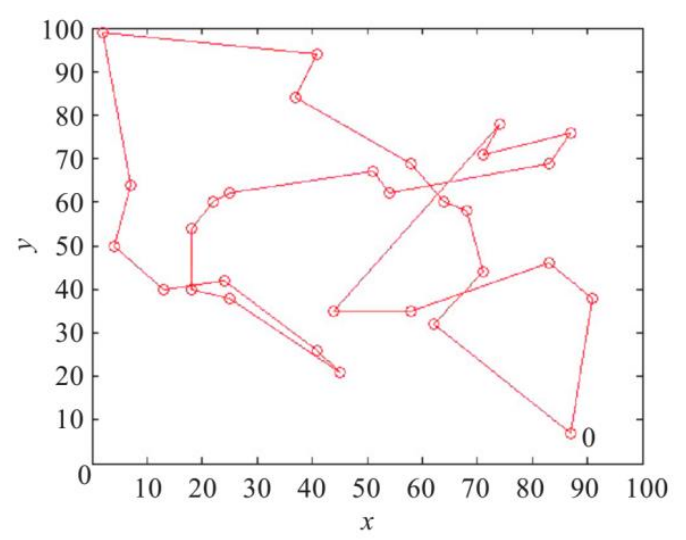

Figure 2. The Path Diagram Adopting Adaptive Genetic Algorithm

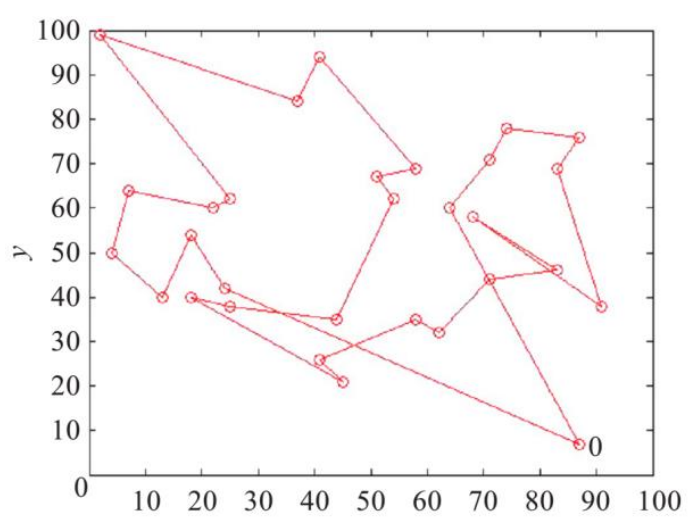

Figure 3. The Path Diagram Adopting Immune Genetic Algorithm

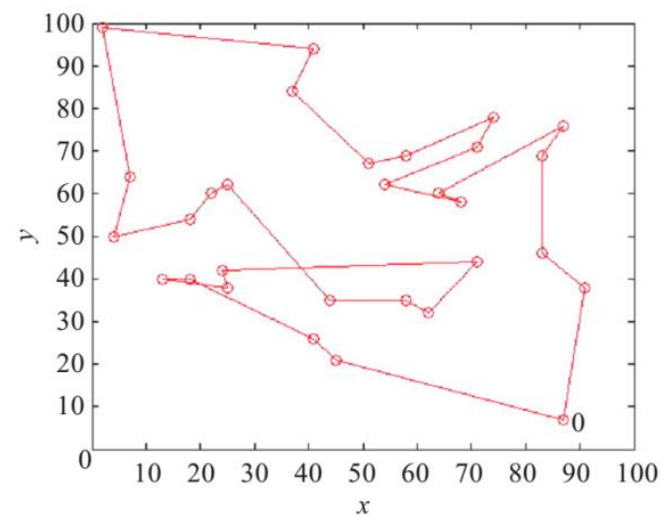

Figure 4. The Path Diagram Adopting Improved Hybrid Algorithm

The path adopting adaptive genetic algorithm is obtained as $0 \rightarrow 1 \rightarrow 2 \rightarrow 28 \rightarrow 27 \rightarrow 8 \rightarrow 9 \rightarrow 7 \rightarrow 6 \rightarrow 11 \rightarrow 12 \rightarrow 18 \rightarrow 17 \rightarrow 19 \rightarrow 22 \rightarrow 24 \rightarrow 26 \rightarrow 25 \rightarrow 23 \rightarrow 21$ $\rightarrow 20 \rightarrow 16 \rightarrow 15 \rightarrow 14 \rightarrow 13 \rightarrow 10 \rightarrow 4 \rightarrow 5 \rightarrow 3 \rightarrow 29 \rightarrow 0$.

The path adopting immune genetic algorithm is obtained as $0 \rightarrow 4 \rightarrow 9 \rightarrow 8 \rightarrow 7 \rightarrow 6 \rightarrow 1 \rightarrow 5 \rightarrow 2 \rightarrow 3 \rightarrow 29 \rightarrow 28 \rightarrow 25 \rightarrow 26 \rightarrow 22 \rightarrow 24 \rightarrow 27 \rightarrow 11 \rightarrow 12 \rightarrow 10 \rightarrow 14$ $\rightarrow 13 \rightarrow 15 \rightarrow 18 \rightarrow 17 \rightarrow 16 \rightarrow 20 \rightarrow 21 \rightarrow 19 \rightarrow 23 \rightarrow 0$.

The path adopting improved hybrid genetic algorithm is obtained as $0 \rightarrow 1 \rightarrow 2 \rightarrow 6 \rightarrow 7 \rightarrow 4 \rightarrow 5 \rightarrow 11 \rightarrow 9 \rightarrow 8 \rightarrow 10 \rightarrow 12 \rightarrow 13 \rightarrow 14 \rightarrow 15 \rightarrow 16 \rightarrow 20 \rightarrow 19 \rightarrow 17 \rightarrow 18$ $\rightarrow 27 \rightarrow 28 \rightarrow 29 \rightarrow 3 \rightarrow 23 \rightarrow 24 \rightarrow 21 \rightarrow 22 \rightarrow 25 \rightarrow 26 \rightarrow 0$. 
By the simulation results, under the situation that the iterations times is 1000 , the path length is 513.7 units using the adaptive genetic algorithm, the time cost is $29 \mathrm{~s}$; the path length is 522.1 units using the immune genetic algorithm, the time cost is $28 \mathrm{~s}$; the path length is 486.2 units using the improved hybrid algorithm, the time cost is $32 \mathrm{~s}$. The obtained data is shown in Table 1 .

Table 1. The Data after 1000 Times of Iteration in Three Kinds of Algorithm

\begin{tabular}{|c|c|c|}
\hline Algorithm classification & The path length & Consumed Time /s \\
\hline Adaptive genetic algorithm & 513.7 & 29 \\
\hline Immune genetic algorithm & 522.1 & 28 \\
\hline Improved hybrid algorithm & 486.2 & 32 \\
\hline
\end{tabular}

It can be seen from the experimental simulation results, under the condition of same iteration times, the path length is the shortest adopting the proposed hybrid algorithm in this paper.

In this paper, due to the combination with $\mathrm{BP}$ algorithm, the improved hybrid algorithm has longer single iteration time, but its solving efficiency is high, the path length is the shortest under the same iteration times. Although the time cost is a little longer than other two algorithms under the same iteration times, in the path planning of logistics activity, based on the issues of cost factor, the path length is the most important evaluation criterion, thus the improved hybrid algorithm is relatively appropriate.

(2) The simulation while path length and time cost are in certain cases

Under the condition of upper simulation data, three kinds of algorithms respectively do path planning, the relation curves among iteration times, path length and time cost are shown in Figure 5, Figure 6 and Figure 7 (in the diagram, curve L1 represents the relationship between path length and iteration times, curve L2 is on behalf of the relationship between time and iteration times).It is shown from Figure 5, Figure 6 and Figure 7, in the case of the solved path length is in certain value (the path length is set as 600 units), the simulation results are as follows: the time cost is $26 \mathrm{~s}$ using the adaptive genetic algorithm, and the iteration times is 897 ; using immune genetic algorithm takes 23 $\mathrm{s}$, the iteration times is 821 ; using improved hybrid algorithm takes $21 \mathrm{~s}$, the iteration times is 657.The data number is shown in Table 2.

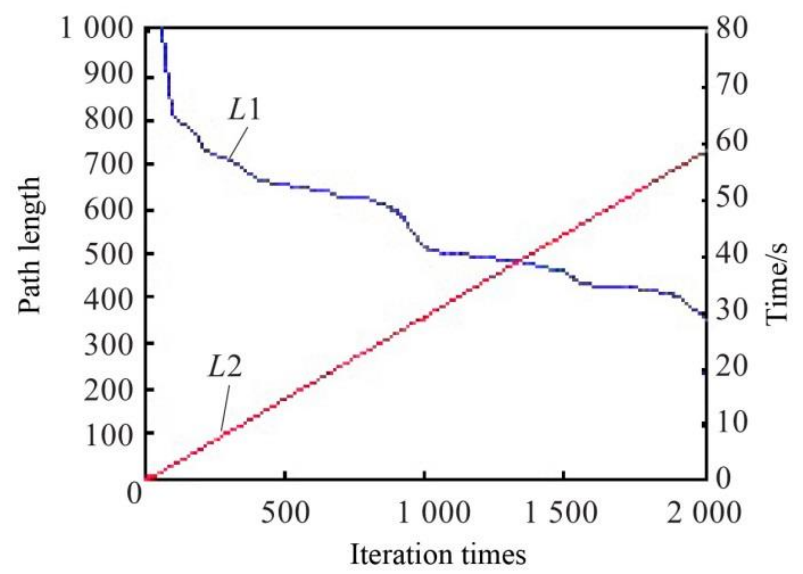

Figure 5. The Iterations Times and Time Cost Adopting Adaptive Genetic Algorithm 


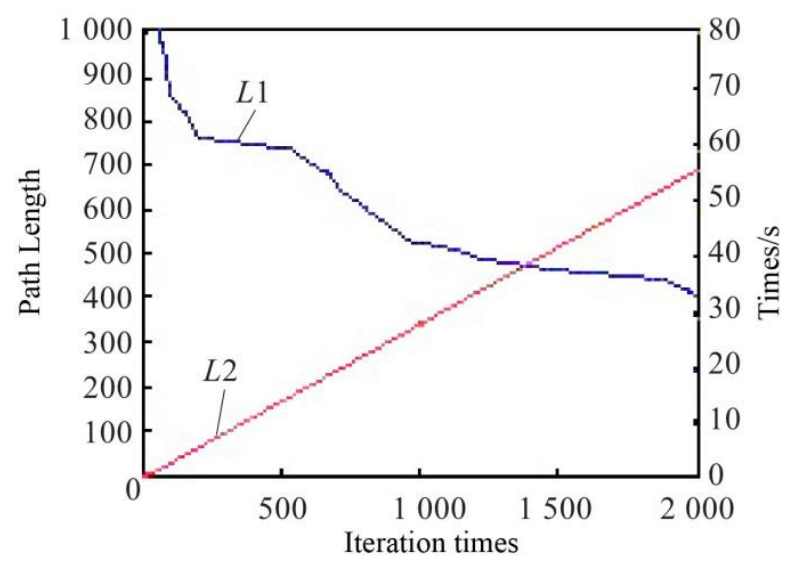

Figure 6. The Iteration Times and Time Cost Adopting Immune Genetic Algorithm

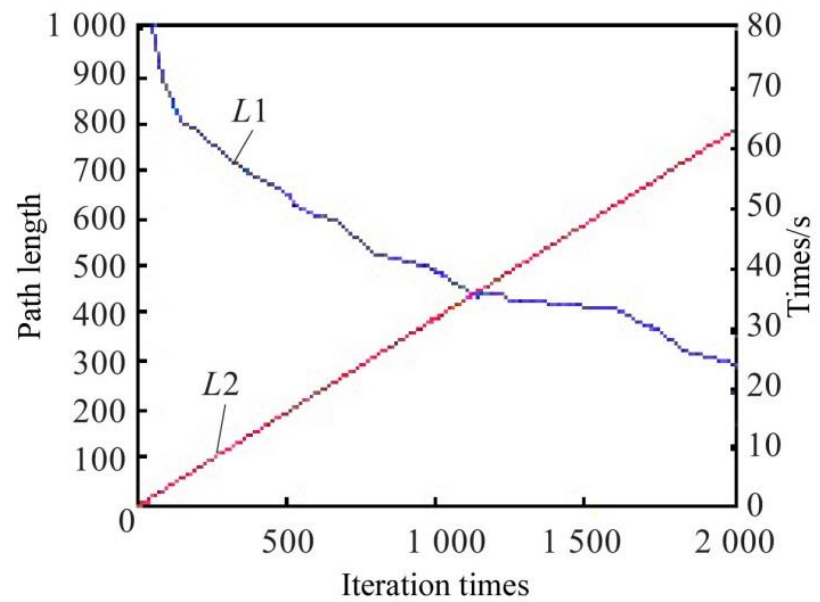

Figure 7. The Iteration Times and Time Cost Adopting Improved Hybrid Algorithm

Table 2. The Obtained Data of Three Algorithms Respectively Reaching to 600 units

\begin{tabular}{|c|c|c|}
\hline Algorithm classification & Iteration times & Time cost /s \\
\hline Adaptive genetic algorithm & 897 & 26 \\
\hline Immune genetic algorithm & 821 & 23 \\
\hline Improved hybrid genetic algorithm & 657 & 21 \\
\hline
\end{tabular}

It is shown from Figure 5, Figure 6 and Figure 7, in the condition with same time cost (it is set as $55 \mathrm{~s}$ ), the simulation results of three kinds of algorithms are as follows: the shortest path length adopting the adaptive genetic algorithm is 403.8 units, the iteration times is 1897 ; the shortest path length adopting the immune genetic algorithm is 419.5 units, the iteration times is 1965 ; the shortest path length adopting the improved hybrid algorithm is 376.1 units, the iteration times is 1, 719.The obtained data is shown in Table 3. 
It is shown from the simulation results, comparing to other two algorithms, under the condition of same path length, the time cost using the improved hybrid algorithm proposed in this paper is the shortest; in the condition with same time cost, the obtained path length using the hybrid algorithm proposed in this paper is the shortest.

Table 3. The Data Calculated by Three Kinds of Algorithms at 55s

\begin{tabular}{|c|c|c|}
\hline Algorithm classification & The shortest path length & Iteration times \\
\hline Adaptive genetic algorithm & 403.8 & 1897 \\
\hline Immune genetic algorithm & 419.5 & 1965 \\
\hline $\begin{array}{c}\text { Improved hybrid genetic } \\
\text { algorithm }\end{array}$ & 376.5 & 1719 \\
\hline
\end{tabular}

\subsection{The Analysis and Evaluation of Experimental Results}

It is shown from the examples simulation, the improved hybrid algorithm proposed in this paper compares with other two kinds of genetic algorithms, the convergence speed is faster, and the accuracy is higher. For the problem solving, the improved hybrid algorithm can find shorter path length than other two kinds of genetic algorithms, the solution quality has great improvement. The improved hybrid algorithm is not only simple in the operation, but also the convergence effect is better, the convergence speed is faster, and the solving quality is higher.

\section{Conclusions}

This paper studies the algorithms of vehicle path planning problem, based on the analysis of the merits and demerits among the genetic algorithms and BP algorithm, develops an improved hybrid algorithm which combines the two kinds of algorithm together, to solve the vehicle routing planning problems in the logistics activity. The characteristic of the method is to generate the next generation of new population by applying the BP algorithm into local optimization, effectively improves the optimization efficiency of genetic algorithm, and then use BP algorithm to do local fine-tuning to obtain the optimal solution. The improved hybrid algorithm can better solve poor local optimization ability of genetic algorithm and the low efficiency of fine adjustment for optimal solution. The adaptive genetic algorithm, the immune genetic algorithm and the improved hybrid algorithm are compared by simulation results in MATLAB environment respectively, the proposed hybrid algorithm in such aspects as optimization ability and efficiency is better than the other two algorithms, in solving the vehicle path planning problem it has the advantage of high speed and high quality, therefore this method is feasible, effective and superior.

\section{Acknowledgements}

This paper is supported by the funds of the science and technology research key project of Henan province (2014), China. The project name is "The scheduling model of logistics vehicles and their optimization based on the dynamic demand", and the project number is 142102210231. 


\section{References}

[1] G. B. Danzig and J. H. Ramseur, “ The truck dispatching problem”, Management Science, vol.6, no.1, (1959), pp. 80-91.

[2] Liuyong Song, Rui Wang and Yongwang Zhou, "Dynamic urban transportation network optimization model and algorithm design", Journal of surveying and mapping science, vol.36, no.1, (2011), pp. 134136.

[3] Yancheng gong, Xiaofen Guo and Xiaoling You, "Research of logistics distribution vehicle scheduling problem based on genetic algorithm", The practice and understanding of mathematics, vol. 34, no.6, (2004), pp. 93-97.

[4] Yiming Chao, "A tabu search method for the truck and trailer routing problem", Computer and Operations Research, vol. 29, no.1, (2002 ), pp.33-51.

[5] Chen Yaorong, Liang Bo and Zhou Meihua, "Optimization for vehicle scheduling in iron and steel works based on semi-trailer swap transport", Journal of Central South University of Technology, vol, 17,no.4, (2007), pp. 873-879.

[6] Shiquan Zhong and Guoguang He, "A tabu algorithm for vehicle scheduling optimization problem with time windows limitation", Journal of systems engineering theory method, vol. 14, no. 6, (2005), pp. 523527.

[7] Luliang Tang, Xiaomeng Change and Qingquan Li, "The public travel path optimization based on ant colony optimization algorithm and the taxi GPS data", China journal of highway and transport, vol. 24, no.2,(2011), pp. 89-95.

[8] Tian Jingwen and Sun Yang, "WSN path optimization based on fusion of improved ant colony algorithm and genetic algorithm", Journal of Computational Information Systems, vol.6, no.5, (2010), pp.1591-1599.

[9] Tian Jingwen and Gao Meijuan, "Web text mining based on improved genetic algorithm and radial basis function neural network", Journal of Computational Information Systems, vol.8, no.3, (2012), pp. 1195-1202.

[10] Chengbo Li, Xiaoming Wang and Qiang Liu, "WMSNS multipath multi-objective optimization algorithm based on genetic algorithm", Computer application research, vol.29, no.6, (2012), pp. 22772282.

[11] Lin Lei, Weifeng Li and Houjun Wang, "Wireless sensor network path optimization based on genetic algorithm", Journal of university of electronic science and technology, vol.38, no. 2, (2009), pp. 227-230.

[12] Kaibing Yang and Xiaobing Liu, "The improved Pareto adaptive genetic algorithm to solve the multiobjective combinatorial optimization", Computer engineering and application, vol. 45, no.8, (2009), pp. 44-46.

[13] Yanchu Yang, Sheng Wang and YidongGu, "The near space airship multiple disciplinary optimization design based on the genetic algorithm", Computer simulation, vol. 29, no. 4, (2012), pp. 49-54.

[14] XiaohuaLi, Zhengyu Zhu and Mengshuang Xia, "Multiple-depot CARP problem via improved genetic algorithm", Computer engineering and application, vol.45, no. 11, (2009), pp. 230-234.

[15] Yingxin Chen, "Vehicle routing optimization problem based on improved ant colony algorithm", Computer application research, vol.29, no.6, (2012), pp. 2031-2034.
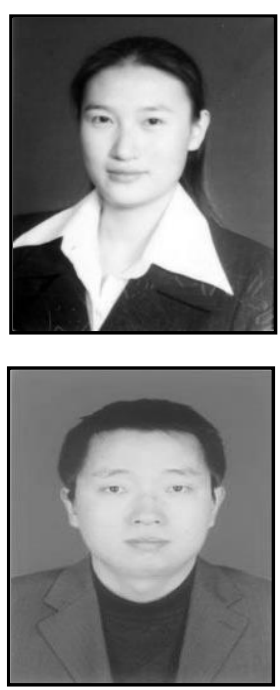

\section{Authors}

Lingxia Liu, She was born in 1976.5, she incepted her master degree in Shanxi University in 2003, and her major is information technology. Now she is working in Computer Education Department of Anyang Normal University as an associate professor. She is also a member of China Computer Federation.

Qiang Song, He was born in 1971.2, he incepted his double master degrees in Ateneo de Manila University in the Philippines in 2005, which are MSIT and MBA. Now he is a PhD. Candidate in Wuhan University of Technology in major of information engineering, China. $\mathrm{He}$ is working in Anyang Institute of Technology as a Professor, and he is also a senior member of China Computer Federation. 